\title{
Can hadronic rescattering explain the "jet quenching” at relativistic energies?
}

\author{
David Hardtke \\ Department of Physics, University of California, Berkeley, California 94720 USA \\ Thomas J. Humanic \\ Department of Physics, Ohio State University, Columbus, Ohio 43210 USA \\ (Received 25 May 2004; published 15 March 2005; publisher error corrected 21 March 2005)
}

\begin{abstract}
Recent relativistic heavy-ion collider (RHIC) data have shown novel nuclear modifications of moderate- to high- $p_{T}$ particle production in central $\mathrm{Au}+\mathrm{Au}$ collisions, including a suppression of hadron production and a disappearance of back-to-back hadron pairs. In this paper, we investigate whether final-state hadronic interactions of the jet fragments can reproduce the RHIC data. We find that hadronic rescattering can account for the disappearance of back-to-back hadron pairs but cannot reproduce other features of the RHIC data.
\end{abstract}

DOI: 10.1103/PhysRevC.71.034906

PACS number(s): $25.75 .-\mathrm{q}$

Recent data on the production of high- $p_{T}$ hadrons in central $\mathrm{Au}+\mathrm{Au}$ collisions at the relativistic heavy-ion collider (RHIC) indicate novel nuclear effects. Above $p_{T}=5-6 \mathrm{GeV} / c$, the yield of hadrons is suppressed by a factor of $\sim 5$ compared to what would be expected from an incoherent superposition of an inelastic nucleon-nucleon collision [1,2]. Data on two-particle azimuthal correlations show similar near-angle jetlike correlations in central $\mathrm{Au}+\mathrm{Au}$ and $p+p$ collisions $[3,4]$. The back-to-back dihadrons indicative of dijet production, however, are absent or greatly suppressed in the most central $\mathrm{Au}+\mathrm{Au}$ collisions. In addition, the production of high- $p_{T}$ hadrons shows a strong azimuthal correlation with respect to the reaction plane ("elliptic flow") [3].

Taken together, these experimental data are thought to result from a novel nuclear effect known as jet quenching. Recent measurements from $d+$ Au collisions do not show the same behavior as central $\mathrm{Au}+\mathrm{Au}$ collisions [5], so the modification of moderate- to high- $p_{T}$ hadron production observed in central $\mathrm{Au}+\mathrm{Au}$ collisions is thought to arise primarily from the interaction of fast partons or their fragmentation products with the dense medium produced in central collisions of heavy nuclei. The goal of the current work is to investigate whether the data can be explained entirely in terms of the hadronic interactions of jet fragmentation products with a dense hadronic medium.

Fast partons traversing a dense gluonic medium are expected to lose energy and acquire transverse momentum relative to their original direction of propagation [6-8]. This energy loss is due to radiative induced gluon emission. The rate of energy loss is proportional to the gluon density of the medium traversed. The previously mentioned RHIC data have been described quite successfully by convoluting expected parton production rates, parton energy loss in an expanding dense medium, and parton fragmentation.

To quantify the nuclear matter effects on high- $p_{T}$ particle production, the nuclear modification factor $R_{A A}$ is constructed,

$$
R_{A A}=\frac{d^{2} N^{A A} / d_{p_{T}} d \eta}{T_{A A} d^{2} \sigma^{N N} / d p_{T} d \eta},
$$

where the nuclear overlap function $T_{A A}=\left\langle\mathrm{N}_{\text {bin }}\right\rangle / \sigma_{\text {inel }}^{N N}$ from a Glauber calculation accounts for the nuclear collision geometry. In the absence of nuclear matter effects, $R_{A A}$ should approach unity at moderate $p_{T}(2-3 \mathrm{GeV} / c)$. In central $\mathrm{Au}+\mathrm{Au}$ collisions at $\mathrm{RHIC}, R_{A A} \approx 1 / 5$. This behavior was predicted by models that incorporate partonic energy loss in a dense gluonic medium. A recent paper, however, pointed out that this suppression of single inclusive particle production at high $p_{T}$ can also be explained qualitatively by assuming that partons fragment inside a dense hadronic medium [9]. The basic feature of the single inclusive data $\left(R_{A A} \approx 1 / 5\right)$ is reproduced by this model, although it may fail to describe the exact $p_{T}$ dependence of the suppression. Adopting the notion of a colorless prehadron allows for a better description of the $p_{T}$ dependence of $R_{A A}$ [10]. With suitable modifications to the initial-state nuclear effects, it is likely that this hadronic rescattering model could give a reasonably qualitative description of the single inclusive data, including the exact $p_{T}$ dependence of $R_{A A}$.

The ability to describe the single inclusive data in terms of both parton energy loss in a dense gluonic medium and hadronic rescattering and energy loss in a dense hadronic medium is not surprising. Any many-body calculation based on the Boltzmann equation yields identical single-particle distributions under the substitution $\sigma \rightarrow A \sigma$ and $\rho \rightarrow \rho / A$, where $\sigma$ is the two-body scattering cross section, $\rho$ is the density of scattering centers, and $A$ is an arbitrary number. Thus, a single-particle observable is unable to distinguish between a dense medium with small scattering cross sections and a more dilute medium with larger scattering cross sections [11]. In general, QCD energy loss cannot be described using the Boltzmann equation as QCD energy loss processes may be coherent. Nonetheless, the uncertainties in the dynamical evolution of a heavy-ion collision coupled with the uncertainties in nuclear effects in parton production from cold nuclei make it difficult to distinguish between the partonic and hadronic energy loss scenarios using single-particle data alone.

Fluctuation observables are not invariant, however, under the substitution $\sigma \rightarrow A \sigma$ and $\rho \rightarrow \rho / A$. For $A>1$, fluctuations will be increased. Fluctuations can be measured via many observables but are most easily quantified in terms of two-particle correlations. The production of jets in a heavy-ion collision can be thought of in terms of a local fluctuation 


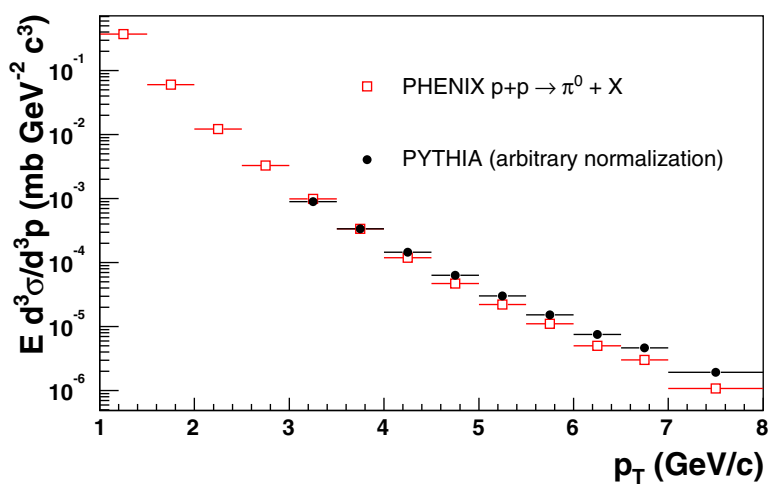

FIG. 1. (Color online) Comparison of the invariant spectra from RHIC $p+p$ data [13] and PYTHIA. The PYTHIA spectrum has been arbitrarily normalized.

in high- $p_{T}$ particle production. In this paper, we investigate what a hadronic rescattering interpretation of the RHIC single inclusive particle production data would predict for two-particle azimuthal correlations. We find that the hadronic rescattering picture is unable to simultaneously describe the RHIC data on inclusive particle suppression and high- $p_{T}$ azimuthal correlations. The paper is organized as follows. We first compare RHIC data directly to the PYTHIA event generator and find that this model reasonably describes the features of moderate- to high- $p_{T}$ inclusive particle production and two-particle azimuthal correlations. We then introduce a hadronic rescattering model that will be used to model a dense hadronic system into which we will embed fragmentation products from PYTHIA events. After merging the PYTHIA jet events with the rescattering model, we study the propagation of PYTHIA fragmentation products in our dense hadronic medium. We find quantitative disagreement with the RHIC data, particularly because of the copious resonance production from hadronic rescattering that should be manifest in the two-particle azimuthal correlations but is not observed in the experimental data.

\section{THE PYTHIA EVENT GENERATOR: COMPARISON TO RHIC DATA}

To model hard scattering processes and fragmentation, we use the PYTHIA event generator [12]. This model convolutes measured parton distribution functions, elementary parton-parton scattering cross sections, and a phenomenological model of jet fragmentation. In Fig. 1 we compare the invariant $p_{T}$ spectra for pions produced running PYTHIA with its standard settings with the recently measured PHENIX $p+p$ data [13]. For our studies here, we are only concerned that the shape of the PYTHIA spectra is similar to the data, so the PYTHIA spectrum is normalized to match the real data at $p_{T}=4 \mathrm{GeV} / c$. As seen, the PYTHIA event generator with default settings does a reasonable job in describing the measured RHIC data between 4 and $8 \mathrm{GeV} / c$.

Our main goal in this paper is to investigate azimuthal correlations among high- $p_{T}$ hadrons. In Fig. 2, we compare the azimuthal correlations measured by the STAR Collaboration

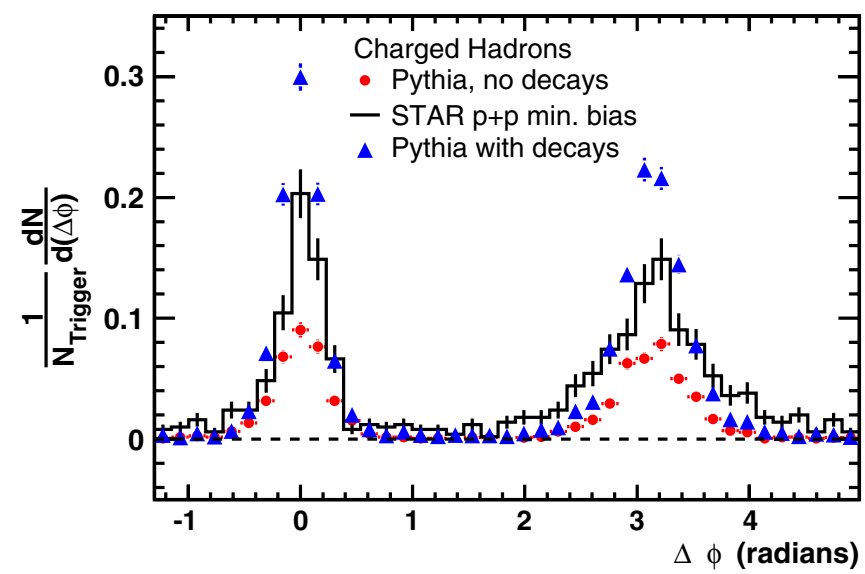

FIG. 2. (Color online) Per-trigger-hadron relative azimuthal distributions for STAR data [4] compared to PYTHIA calculations with and without resonance decays.

[4] to those produced by the PYTHIA event generator. The PYTHIA calculations use kinematic cuts identical to those used by the STAR Collaboration (pseudorapidity $\eta<0.7$ ). Events with a high- $p_{T}$ trigger hadron with $4<p_{T}<6 \mathrm{GeV} / c$ are found. We then calculate the azimuthal separation of other hadrons in these events with $p_{T}>2 \mathrm{GeV} / c$. The resulting azimuthal distribution is normalized to the number of trigger hadrons. Both the STAR data and the PYTHIA calculations yield strong azimuthal correlations near $\Delta \phi \approx 0$ and $\Delta \phi \approx \pi$. The correlated back-to-back hadrons arise from the fragmentation of back-to-back dijets.

The correlated small-angle pairs can be produced via two mechanisms. During jet fragmentation, the produced hadrons will be collimated, thereby producing azimuthally correlated particle pairs. Such correlated pairs, however, can also be the result of the decay of resonances. In Fig. 2 we investigate the role of resonance decays in the PYTHIA calculations. Within this model, stable hadrons $(\pi, K, p)$ are produced directly during string fragmentation and also during the decay of unstable resonances produced during string fragmentation. Figure 2 shows two different PYTHIA calculations. The standard PYTHIA setting includes decay of unstable resonances, and we see a strong near-angle and back-to-back correlation peak. We also ran PYTHIA in a mode where no resonances produced during fragmentation were allowed to decay. In this mode nearangle and back-to-back correlations are also observed. These correlations, however, are greatly suppressed. It is important to note, however, that the suppression factor is identical for the near-angle and back-to-back hadron pairs. Since the back-toback hadron pairs cannot arise from low mass resonance decay (the invariant mass of the parent would need to be greater than $5 \mathrm{GeV}$ ), we conclude that the near-angle correlations observed in PYTHIA arise primarily from the jet fragmentation and are not due to resonance decay. Turning on the resonance decay leads to the observation of more charged hadrons per event and hence increases the strength of the near-angle and back-to-back azimuthal correlations. Analogously, if we were to include $\pi^{0}$ in the construction of the azimuthal distribution, we would see a trivial $50 \%$ increase in the near-angle and back- 
to-back correlation strength. The increase of the azimuthal distributions is due primarily to the observation of the extra hadrons from resonance decay and not from the correlations induced by these decays.

It should be noted that the absolute per-trigger yield of associated particles differs between the PYTHIA and STAR data. In the case of PYTHIA with decays, the per-trigger yield of associated particles is larger for PYTHIA than for STAR data. In the case of PYTHIA without decays, the yield is smaller than for the STAR data. No attempt is made to adjust the PYTHIA fragmentation settings to rectify this difference. These azimuthal distributions are normalized to the number of trigger hadrons, and these mostly arise from the case where the leading hadron carries a large fraction of the parton momentum [14]. This is the region where the experimental data on parton fragmentation functions are most uncertain and the PYTHIA fragmentation functions are tuned to experimental data. Thus, any discrepancy between measured dihadron correlation data and the PYTHIA calculations is not of fundamental concern.

\section{A SIMPLE HADRONIC RESCATTERING MODEL}

The hadronic rescattering model [15] that we will use to understand high- $p_{T}$ hadron propagation in a dense hadronic medium has been described elsewhere and shown to reproduce many of the low- $p_{T}$ experimental observables at RHIC including transverse momentum distributions for $\pi, K, p, v_{2}\left(p_{T}\right)$ and the Hanbury-Brown Twiss radii [16]. We briefly review some of the features of the model below.

Rescattering is simulated with a semiclassical Monte Carlo calculation which assumes strong binary collisions between isospin-averaged hadrons. Relativistic kinematics is used throughout. All calculations are made to simulate $\mathrm{RHIC}$-energy $\mathrm{Au}+\mathrm{Au}$ collisions in order to compare with the results of RHIC data.

The initial stage of the rescattering calculation employs simple parametrizations to describe the initial momenta and space time of the hadrons. The initial momenta are assumed to follow a thermal-like transverse momentum distribution for all particles,

$$
\left(1 / m_{T}\right) d N / d m_{T}=C m_{T} /\left[\exp \left(m_{T} / T\right) \pm 1\right],
$$

where $T$ is a temperature parameter, and a Gaussian rapidity distribution for mesons,

$$
d N / d y=D \exp \left[-\left(y-y_{0}\right)^{2} /\left(2 \sigma_{y}^{2}\right)\right],
$$

where $\sigma_{y}$ is the rapidity width. Two rapidity distributions for baryons have been tried: (i) flat and then falling off near beam rapidity and (ii) peaked at central rapidity and falling off until beam rapidity. Both baryon distributions give about the same results. The initial longitudinal particle hadronization position $z_{\text {had }}$ and time $t_{\text {had }}$ are determined by the relativistic equations,

$$
z_{\text {had }}=\tau_{\text {had }} \sinh y, \quad t_{\text {had }}=\tau_{\text {had }} \cosh y,
$$

where $\tau_{\text {had }}$ is the hadronization proper time. From Eqs. (2) and (3), it is seen that longitudinal invariance is not assumed in the initial conditions for the present calculations. Calculations were carried out using isospin-summed events containing at

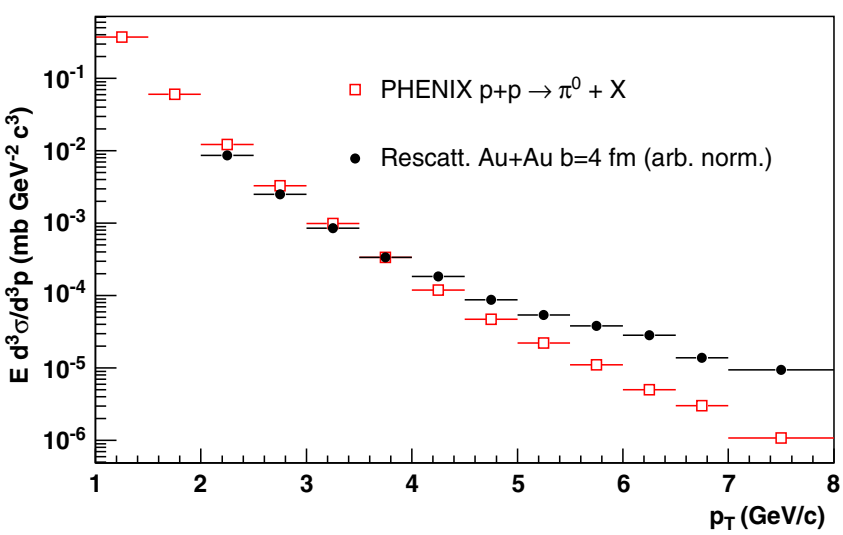

FIG. 3. (Color online) Comparison of the invariant spectra from RHIC $p+p$ data and a pure rescattering model calculation for $\mathrm{Au}+\mathrm{Au}$ collisions with $b=4 \mathrm{fm}$. The rescattering model spectra have been arbitrarily normalized.

freeze-out about 5000 pions, 500 kaons, and 650 nucleons ( $\Lambda$ 's were decayed). The hadronization model parameters that were found to reproduce the RHIC data were $T=300 \mathrm{MeV}$, $\sigma_{y}=2.4$, and $\tau_{\text {had }}=1 \mathrm{fm} / c$.

We now compare RHIC high- $p_{T}$ data to calculations from the pure rescattering model. In other words, for these calculations hard scattering and fragmentation are not implemented. In Fig. 3 we compare the PHENIX $p+p \rightarrow \pi^{0}+X$ data to the pure rescattering model calculations for $\mathrm{Au}+\mathrm{Au}$ collisions with impact parameter $b=4 \mathrm{fm}$. For high- $p_{T} \pi^{0}$ production at RHIC, the spectral shape is similar in central $\mathrm{Au}+\mathrm{Au}$ and $p+p$ collisions. We are only interested in spectral shapes, so the rescattering model calculations are arbitrarily normalized to the PHENIX data at $p_{T}=4 \mathrm{GeV} / c$. (The integral of the $p_{T}$ spectra and hence the total yield is used to adjust the initial conditions of the calculation.) We see that the rescattering model produces copious high- $p_{T}$ hadrons and exhibits a power law behavior. The rescattering model overpredicts the yield of high- $p_{T}$ hadrons. This is probably because the treatment of the densest stage of the collision is in terms of $2 \rightarrow 2$ binary hadronic collisions, whereas a more correct treatment would model this stage using many-body collision dynamics. It has been verified numerically that this approximation does not affect the bulk dynamics of the collision [11] as measured by low- $p_{T}$ observables.

The pure rescattering model calculations also show near-angle (but not back-to-back) azimuthal correlations. Figure 4 compares the measured azimuthal distributions for $p+p$ collisions [4] and the azimuthal correlations produced in the rescattering model calculation for $\mathrm{Au}+\mathrm{Au}$ at $b=4 \mathrm{fm}$. The near-angle correlations in the rescattering model come from the decay of resonances. We confirmed this by considering rescattering particles that do not come directly from a resonance decay, in which case no azimuthal correlations are observed. These correlations are both stronger and broader than those measured in the $p+p$ data. Having fit the rescattering model near-angle azimuthal distribution with a Gaussian, we obtained a width of $\sigma=0.39 \pm 0.04$. In contrast, STAR measured the widths of the near-angle azimuthal correlation in $p+p, d+\mathrm{Au}$, and central $\mathrm{Au}+\mathrm{Au}$ collisions and obtained 


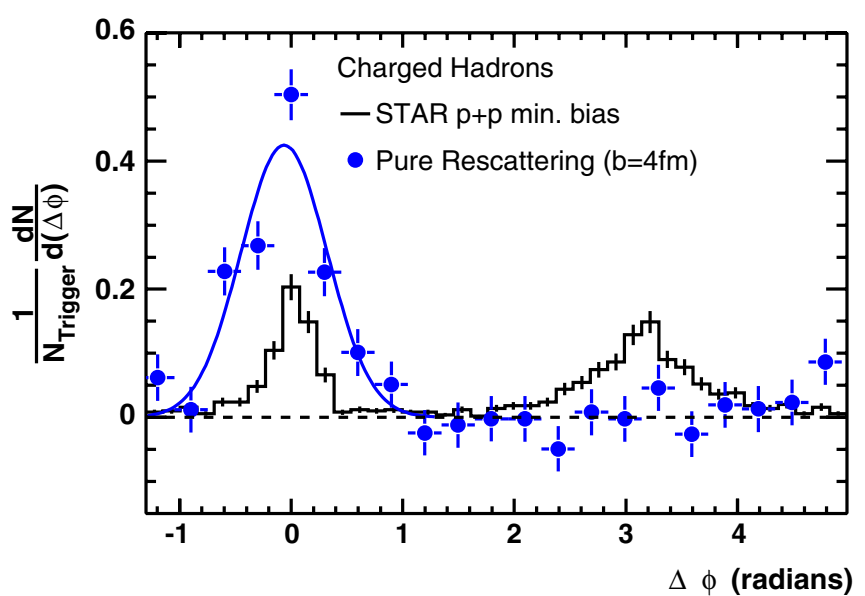

FIG. 4. (Color online) Per-trigger-hadron relative azimuthal distributions for STAR $p+p$ data compared to a pure rescattering model calculation for $\mathrm{Au}+\mathrm{Au}$ at $b=4 \mathrm{fm}$. The blue line shows a Gaussian fit to the azimuthal distribution from the rescattering model.

a width in all cases of $\sigma \approx 0.2$ for these kinematic cuts. The large discrepancy in the widths of these correlations indicates a fundamentally different origin. In the rescattering model, the correlations come from resonance decay, whereas for the RHIC data the correlations seem to arise primarily from jet fragmentation.

The near-angle azimuthal correlation strength is related to the yield of resonances at $p_{T} \approx p_{T}$ (trigger) $+p_{T}$ (associated). The fact that the pure rescattering model produces an invariant $p_{T}$ spectrum of hadrons and resonances that is harder than that measured in the data leads to a somewhat artificial increase in the near-angle correlation strength. This model is certainly incorrect for calculating moderate- to high- $p_{T}$ particle production in $\mathrm{Au}+\mathrm{Au}$ collision at RHIC, so we chose to make no attempt to correct the model for its possible shortcomings. The rescattering model will only be used as a rough estimate of the environment that a jet fragmentation product would see if it were able to fragment inside such a dense hadronic medium. Nonetheless, we note that this pure rescattering model does produce azimuthal correlated particle pairs due to resonance decay and that these correlations are broader and stronger than those seen in the real data.

\section{THE PROPAGATION OF PYTHIA TEST PARTICLES IN THE RESCATTERING MODEL}

PYTHIA events are embedded into these rescattering events, with the production vertices chosen to account for the initial nuclear overlap geometry. The embedded PYTHIA events are required to have a high- $p_{T}$ hadron with $p_{T}>$ $3 \mathrm{GeV} / c$. The particles coming from these PYTHIA events are tagged as such, and any resonance produced via the interaction of a PYTHIA particle is tagged as a PYTHIA resonance. The decay products of these PYTHIA resonances are tagged as PYTHIA particles, and in the plots that follow we only look at the final-state PYTHIA particles. Thus, the artificially large, high- $p_{T}$ particle production seen in the pure rescattering model does not invalidate our subsequent calculations.

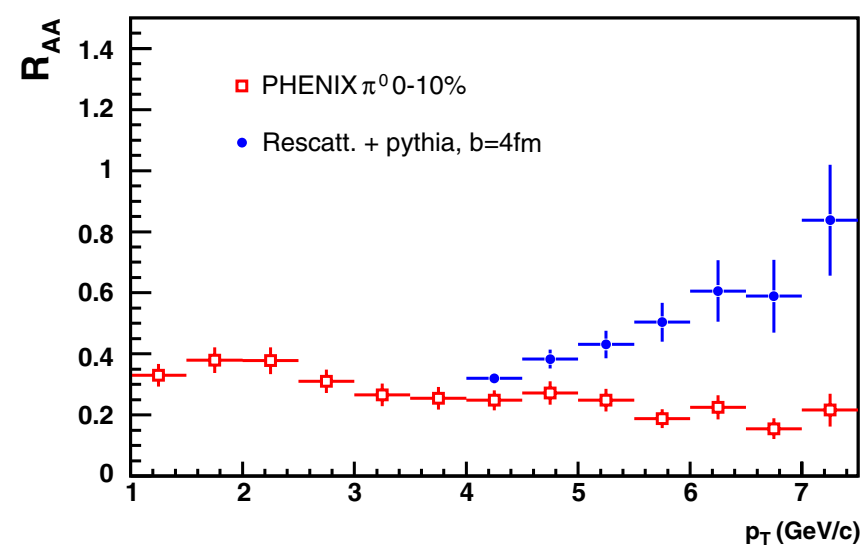

FIG. 5. (Color online) Nuclear modification factor $R_{A A}$ for pions. The open squares show PHENIX $\mathrm{Au}+\mathrm{Au}$ data for the $10 \%$ most central collisions, and the solid circles show the results of the rescattering model with embedded PYTHIA events. Only particles tagged as originating from PYTHIA or a PYTHIA-particle-induced resonance are included in the rescattering+PYTHIA calculation.

The main theoretical uncertainty in these studies is the space-time development of hadrons in parton fragmentation. In the standard PYTHIA fragmentation scheme, no attempt is made to describe the space-time aspects of jet fragmentation. There are many theoretical models of how jet fragmentation develops, but little experimental support for any single idea. In the conventional QCD model, hadrons from parton fragmentation are formed at time $t=E R^{2}$ [17], where $E$ is the parton energy and $R$ is the hadronic size. Thus, very-high- $p_{T}$ hadrons would necessarily form well outside the medium produced in a $\mathrm{Au}+\mathrm{Au}$ collision at RHIC. In another picture, colorless prehadrons are formed very early [18], in which case hadrons would form deep inside the medium produced at RHIC. The prehadrons form instantly in the limit where $z_{h} \rightarrow 1$, where $z_{h}$ is the fraction of the parton momentum carried by the leading hadron. One argument against such a hadron formation picture at RHIC is that hadrons should not exist in a quark-gluon plasma. The instantaneous formation of a colorless prehadron is based on the notion of vacuum energy loss and motivated by the string picture. In a quark-gluon plasma, Debye screening leads to the modification of the string tension and would reduce the rate of vacuum energy loss.

Here we adopt a naive picture of hadron formation, investigate its consequences, and rule it out along with any similar fragmentation picture. In these calculations, the hadrons from the PYTHIA events initially form at proper time $\tau$ and at $(z, t)$ as given by Eq. (4) with $\tau_{\text {had }}=\tau=1 \mathrm{fm} / c$, to be consistent with the rest of the calculation. In the transverse direction, the $(x, y)$ position of the embedded PYTHIA events are randomly selected using the nuclear overlap model. The individual hadrons in each PYTHIA event are further smeared in a circle of radius $1.5 \mathrm{fm}$. This radius is determined by the condition that it is large enough such that hadronic rescattering within a PYTHIA event is small.

Figure 5 shows the nuclear modification factor $R_{A A}$ for pions from these calculations as well as PHENIX $\mathrm{Au}+\mathrm{Au}$ data 


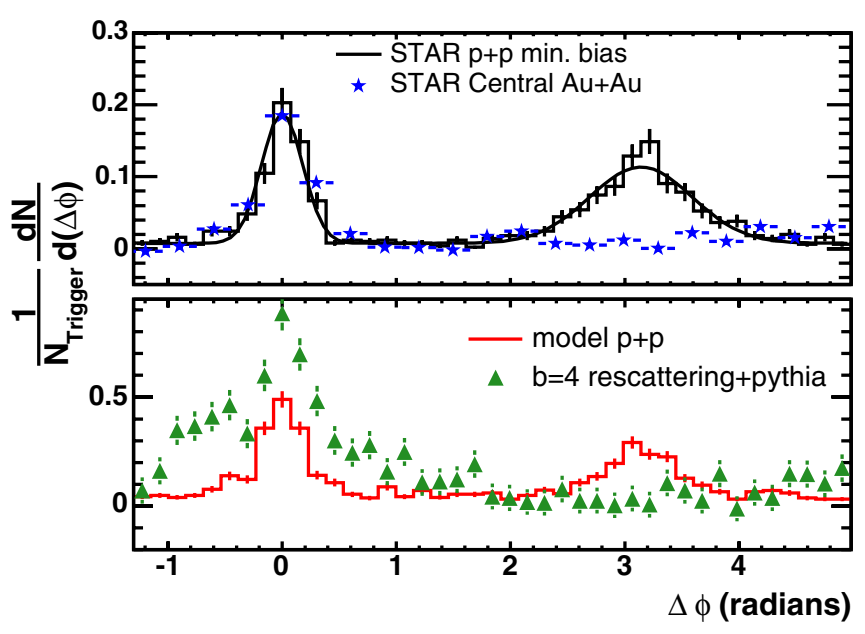

FIG. 6. (Color online) Per-trigger-hadron relative azimuthal distributions for STAR data compared to PYTHIA+rescattering model calculations. Only particles tagged as originating from PYTHIA or a PYTHIA-particle-induced resonance are included in the rescattering+PYTHIA calculation.

[1] at a similar impact parameter. For the model calculations, $R_{A A}$ is defined as

$$
R_{A A}\left(p_{T}\right)=\frac{N(\text { PYTHIA tagged from rescattering events })}{N(\text { PYTHIA } p+p)} .
$$

With this definition, we consider only the modification of the spectra of the embedded PYTHIA events and do not consider the contribution to particle production coming from the rescattering model. Because we inject triggered PYTHIA events into the rescattering model, we only consider $R_{A A}$ for $p_{T}>4 \mathrm{GeV} / c$.

Figure 5 shows similar features to the calculations of Gallmeister et al. [9]. The absolute value of $R_{A A}$ is well reproduced in the moderate- $p_{T}$ region $\left(p_{T} \approx 4 \mathrm{GeV} / c\right)$, but $R_{A A}$ trends upward with increasing $p_{T}$, a trend not seen in the experimental data. The $p_{T}$ dependence arises from our treatment of the space-time development of hadrons in jet fragmentation. Higher $p_{T}$ hadrons are not able to interact until proper time $\tau$ and they thus travel a distance of $\gamma \tau$ in the laboratory frame (here $\gamma$ is the Lorentz factor).

Figure 6 shows the two-particle azimuthal distributions from the STAR Collaboration [4] and from the rescattering events with embedded PYTHIA jets. For the model calculations, we only look at hadrons produced directly from the PYTHIA event or produced via the decay of PYTHIA-particleinduced resonances. In the model calculations, all hadrons are considered (charged and neutral), whereas for the STAR measurements only charged hadrons are used. This should lead to $\sim 50 \%$ stronger correlations in the model compared to the real data.

As pointed out by STAR, the correlations near $\Delta \phi \approx 0$ are indicative of jet fragmentation and are very similar in magnitude and width for proton-proton and central $\mathrm{Au}+\mathrm{Au}$ collisions. The azimuthally back-to-back dihadron pairs $(\Delta \phi \approx \pi)$, indicative of dijet production, are present in the proton-proton collisions but absent in the most central $\mathrm{Au}+\mathrm{Au}$ collisions. This feature is well reproduced in the rescattering model plus PYTHIA jet calculations.

Focusing, however, on the region $\Delta \phi \approx 0$ reveals a striking difference between the rescattering + PYTHIA calculations and the STAR data. While the STAR data show little or no modification of the correlation structure in central $\mathrm{Au}+\mathrm{Au}$ collisions compared to the proton-proton reference, the rescattering model leads to a broadening and enhancement of the near-angle correlations. This is exactly the structure observed in the pure rescattering model calculations (where no PYTHIA jets were embedded into the events). These correlations are primarily caused by the resonances produced during the rescattering stages of the collisions and do not come from the initial jet fragmentation. We thus conclude that the near-angle correlation structure observed by STAR in the central $\mathrm{Au}+\mathrm{Au}$ collision is due to jets that fragment outside the medium. If the jets were to fragment inside a dense hadronic medium they would produce resonances that lead to broad azimuthal correlations. The broad-resonanceinduced correlations seen in the PYTHIA+rescattering calculations are not seen in the central STAR $\mathrm{Au}+\mathrm{Au}$ data.

\section{CONCLUSIONS}

The failure of this model to describe in detail all aspects of high- $p_{T}$ particle production at RHIC is not surprising. It is unlikely that a $2 \rightarrow 2$ hadronic scattering description is valid in the early stages of a RHIC collision. For low- $p_{T}$ observables such as elliptic flow and Hanbury-Brown Twiss radii, this model does seem to work. At high $p_{T}$, however, we show that this model does not work, and we feel that no purely hadronic model can describe the "jet quenching" observables seen in central relativistic heavy-ion collisions. The nuclear suppression factor will have an unwanted $p_{T}$ dependence. In addition, the unavoidable copious resonance production will lead to substantial modification in the two-particle high- $p_{T}$ azimuthal correlations. In particular, we find that resonance decays lead to broader high- $p_{T}$ azimuthal correlations, and there is no evidence in the RHIC data for large broadening of the azimuthal correlations. In general, we find that only correlation and fluctuation measurements, of which high- $p_{T}$ azimuthal correlations are one example, are able to distinguish between a purely hadronic description of RHIC data and an interpretation in terms of novel forms of nuclear matter such as the quark-gluon plasma.

\section{ACKNOWLEDGMENTS}

We would like to thank Michael Lisa for help with the manuscript and useful discussions. We thank the staff of the Ohio Supercomputing Center, where these calculations were made. This work was supported by the National Science Foundation under Grant Nos. PHY-0099476 and PHY-0203111. 
[1] K. Adcox et al., Phys. Rev. Lett. 88, 022301 (2002); S. S. Adler et al., ibid. 91, 072301 (2003).

[2] C. Adler et al., Phys. Rev. Lett. 89, 202301 (2002); J. Adams et al., ibid. 91, 172302 (2003).

[3] C. Adler et al., Phys. Rev. Lett. 90, 032301 (2003).

[4] C. Adler et al., Phys. Rev. Lett. 90, 082302 (2003).

[5] J. Adams et al., Phys. Rev. Lett. 91, 072304 (2003).

[6] M. Gyulassy and M. Plümer, Phys. Lett. B243, 432 (1990).

[7] X. N. Wang and M. Gyulassy, Phys. Rev. Lett. 68, 1480 (1992).

[8] R. Baier, D. Schiff, and B. G. Zakharov, Ann. Rev. Nucl. Part. Sci. 50, 37 (2000).

[9] K. Gallmeister, C. Greiner, and Z. Xu, Phys. Rev. C 67, 044905 (2003)
[10] W. Cassing, K. Gallmeister, and C. Greiner, Nucl. Phys. A735, 277 (2004).

[11] T. J. Humanic, nucl-th/0301055.

[12] T. Sjöstrand et al., Comp. Phys. Commun. 135, 238 (2001).

[13] S. S. Adler et al., Phys. Rev. Lett. 91, 182301 (2003).

[14] M. Jacob and P. V. Landshoff, Nucl. Phys. B113, 395 (1976).

[15] T. J. Humanic, Phys. Rev. C 57, 866 (1998).

[16] T. J. Humanic, Nucl. Phys. A715, 641 (2003); nucl-th/0301055.

[17] Yu. L. Dokshitzer, V. A. Khose, A. H. Mueller, and S. I. Troyan, Basics of Perturbative QCD (Editions Frontieres, Gif-sur-Yvette, France, 1991).

[18] B. Z. Kapeliovich, J. Nemchik, E. Predazzi, and A. Hayashigaki, Nucl. Phys. A740, 211 (2004). 